\title{
Inelastic neutron scattering studies of ${ }^{76} \mathrm{Ge}$ and ${ }^{76} \mathrm{Se}$ : relevance to neutrinoless double- $\beta$ decay
}

\author{
B. P. Crider $^{1}$, E. E. Peters ${ }^{2}$, T. J. Ross ${ }^{1,2}$, M. T. McEllistrem ${ }^{1}$, F. M. Prados-Estévez ${ }^{1,2}$, J. M. Allmond ${ }^{3}$, J. R. Vanhoy ${ }^{1,2,4}$ and $^{12 a}$ \\ S. W. Yates ${ }^{1,2 a}$ \\ ${ }^{1}$ Department of Physics and Astronomy, University of Kentucky, Lexington, KY40506-0055 USA \\ ${ }^{2}$ Department of Chemistry, University of Kentucky, Lexington, KY 40506-0055 USA \\ ${ }^{3}$ Joint Institute of Nuclear Physics and Applications, Oak Ridge National Laboratory, Oak Ridge, TN 37831 USA \\ ${ }^{4}$ Department of Physics, United States Naval Academy, Annapolis, MD 21402 USA
}

\begin{abstract}
Inelastic neutron scattering measurements were performed at the University of Kentucky Accelerator Laboratory on enriched ${ }^{76} \mathrm{Ge}$ and ${ }^{76} \mathrm{Se}$ scattering samples. From measurements at incident neutron energies from 2.0 to $4.0 \mathrm{MeV}$, many new levels were identified and characterized in each nucleus; level lifetimes, transition probabilities, multipole mixing ratios, and other properties were determined. In addition, $\gamma$-ray cross sections for the ${ }^{76} \mathrm{Ge}\left(\mathrm{n}, \mathrm{n}^{\prime} \gamma\right)$ reaction were measured at neutron energies up to $5.0 \mathrm{MeV}$, with the goal of determining the cross sections of $\gamma$ rays in $2040-\mathrm{keV}$ region, which corresponds to the region of interest in the neutrinoless double $\beta$ decay of ${ }^{76} \mathrm{Ge}$. Gamma rays from the three strongest branches from the $3952-\mathrm{keV}$ level were observed, but the previously reported $2041-\mathrm{keV} \gamma$ ray was not. Population cross sections across the range of incident neutron energies were determined for the 3952-keV level, resulting in a cross section of $\sim 0.1$ $\mathrm{mb}$ for the 2041-keV branch using the previously determined branching ratios. Beyond this, the data from these experiments indicate that previously unreported $\gamma$ rays from levels in ${ }^{76} \mathrm{Ge}$ can be found in the $2039-\mathrm{keV}$ region.
\end{abstract}

\section{Introduction}

\subsection{Nuclear structure of the stable $A=76$ nuclei}

The ${ }^{76} \mathrm{Ge}$ and ${ }^{76} \mathrm{Se}$ nuclei lie between the magic numbers of 28 and 50, and shape coexistence plays a prominent role in their structure [1]. Recently, it was proposed by Toh et al. [2] that ${ }^{76} \mathrm{Ge}$ may be a rare example of a nucleus exhibiting rigid triaxial deformation in its lowlying states, i.e., it follows the rigid triaxial model of Davydov and Filipov [3] with a well-defined potential minimum at a non-zero value of $\gamma$. The defining feature on which this claim is based is the energy staggering in the $\gamma$ band. Motivated by this experimental result, Nikšić and coworkers [4] performed calculations within the framework of nuclear density functional theory for the ${ }^{72-}$ ${ }^{82} \mathrm{Ge}$ isotopes. Their analysis did not confirm the evidence for rigid triaxial deformation at low energy in ${ }^{76} \mathrm{Ge}$; in fact, they arrived at the conclusion that the meanfield potential of ${ }^{76} \mathrm{Ge}$ is $\gamma$ soft, more in keeping with the $\gamma$-unstable rotor model of Wilets and Jean [5]. For these reasons, a detailed study of the nuclear structure of this nucleus is easily justified. At the same time, measurements which lead to a characterization of the low-lying level schemes of ${ }^{76} \mathrm{Ge}$ and ${ }^{76} \mathrm{Se}$ can also supply structural information relevant to the calculation of the nuclear matrix elements for the neutrinoless double- $\beta$ decay $(0 v \beta \beta)$ rate.

\subsection{Neutrinoless double- $\beta$ decay}

The observation of neutrino oscillations has revealed that neutrino flavors mix and neutrinos have mass; however, oscillation experiments yield only information on $(\Delta \mathrm{m})^{2}$, and thus the absolute mass scale remains unknown [6]. Double- $\beta$ decay with the emission of two $\beta^{-}$particles and two electron antineutrinos is among the rarest forms of radioactive decay and has been observed for only a handful of nuclei. Neutrinoless double- $\beta$ decay, $0 v \beta \beta$, a leptonnumber-violating nuclear process that has not yet been observed, will occur only if the neutrinos have mass and are Majorana particles, i.e., they are their own antiparticles. The observation of $0 v \beta \beta$ provides the best method for obtaining the mass of the neutrino, and it is the only practical way to establish if neutrinos are Majorana particles. The rate of $0 v \beta \beta$, if driven by the exchange of light Majorana neutrinos, is approximately

$$
\left(T_{0 v}\right)^{-1}=G_{0 v}\left(\mathrm{Q}_{\beta \beta}, \mathrm{Z}\right)\left|M_{0 v}\right|^{2}\left\langle m_{\beta \beta}\right\rangle^{2},
$$

\footnotetext{
a Corresponding author: yates@uky.edu
} 
where $G_{0 v}\left(\mathrm{Q}_{\beta \beta}, \mathrm{Z}\right)$ is the known phase-space factor for the emission of the two electrons, $\left\langle m_{\beta \beta}\right\rangle$ is the effective Majorana mass of the electron neutrino, and $M_{0 v}$ is a nuclear matrix element which must be calculated from nuclear structure models. A focus of many of our recent measurements has been to provide the detailed nuclear structure data for guiding these model calculations.

The approaches employed most frequently during the past two decades to calculate $M_{0 v}$ 's for both $v v \beta \beta$ and $0 v \beta \beta$ decay are derivatives of the shell model (e.g., Ref. [7]) or the quasi-particle random-phase approximation (QRPA) [8]. Recently, calculations within the framework of the interacting boson model (IBM-2) have been introduced [9, $10]$.

For a variety of reasons, several $0 v \beta \beta$ searches focus on the decay of ${ }^{76} \mathrm{Ge}$. The use of ${ }^{76} \mathrm{Ge}$ as both the source of the radiation and the detector, for which the technology is well developed, serves to maximize detection sensitivity for the expected rare events. Moreover, ${ }^{76} \mathrm{Ge}$ is the only nuclide with a reported, although widely criticized, $0 v \beta \beta$ half-life, $1.19 \times 10^{25}$ years [11]. Recent measurements by the GERDA collaboration [12] yield a limit for the half-life that does not support the earlier work of Ref. [11]. Limits on the neutrino mass established from the EXO-200 experiment $[13,14]$ also contradict the reported half-life for $0 v \beta \beta$ of ${ }^{76} \mathrm{Ge}[11]$.

Nuclear structure calculations are crucial to our understanding of $0 v \beta \beta$ (if it is observed) and in determining the mass of the neutrino. Data constraining the nuclear model calculations for double- $\beta$ decay become of particular importance as experimental searches for $0 v \beta \beta$, e.g., the MAJORANA and GERDA collaborations, are coming online or are pushing to increasing sensitivities. With colleagues from Yale University, Triangle Universities Nuclear Laboratory (TUNL), the Technische Universität Darmstadt, and the Australian National University, the University of Kentucky group has initiated a diverse program for obtaining detailed spectroscopic data relevant to $0 v \beta \beta$ decay of ${ }^{76} \mathrm{Ge}$.

\subsection{Background Issues in double- $\beta$ decay}

The "signal" of ${ }^{76} \mathrm{Ge} 0 v \beta \beta$ is a single peak at $\mathrm{Q}_{\beta \beta}$, $2039.06 \pm 0.01 \mathrm{keV}[15]$, in the spectrum of the ${ }^{76} \mathrm{Ge}$ enriched HPGe detectors (see Figure 1 [16]). As even weak background $\gamma$ rays occurring near $Q_{\beta \beta}$ can complicate the analysis of $0 v \beta \beta$ experiments, it is important to assess, quantify, and eliminate these possible obstacles [6]. A $2040.70 \pm 0.25 \mathrm{keV} \gamma$ ray has been placed as de-exciting the $3952-\mathrm{keV}$ level in ${ }^{76} \mathrm{Ge}$ [17]. Camp and Foster [17] first established the existence of the $3952-\mathrm{keV}$ level by observing its population in ${ }^{76} \mathrm{Ge}$ following the subsequent $\beta$ decay from ${ }^{76} \mathrm{Ga}\left(\mathrm{T}_{1 / 2}=32.6 \mathrm{~s}\right.$, $\left.\mathrm{J}^{\pi}=\left(2^{+}, 3^{+}\right), \mathrm{Q}_{\beta}=7.0 \mathrm{MeV}\right)$. As shown in Fig. 2, the $3952-\mathrm{keV}$ level is reported to have five de-exciting $\gamma$ rays. Of course, of particular concern is the 2041-keV $\gamma$ ray, which lies very near the ${ }^{76} \mathrm{Ge}$ region of interest for $0 v \beta \beta$ observation. Admittedly, the $3952-\mathrm{keV}$ level is approximately the $70^{\text {th }}$ excited level of ${ }^{76} \mathrm{Ge}$ and the 2041 $\mathrm{keV}$ ray is a $4 \%$ branch, so its intensity is expected to be low. In the tonne-scale experiments envisioned, however, this level could be excited by inelastic scattering of fast neutrons produced by the $(\alpha, n)$ reaction on light elements, where $\alpha$ particles arise from natural radioactivity in the surroundings, or from cosmic-ray muon-induced neutrons. Also, ${ }^{76} \mathrm{Ga}$ can be produced by the ${ }^{76} \mathrm{Ge}(\mathrm{n}, \mathrm{p})$ reaction, but higher-energy neutrons, $\mathrm{E}_{\mathrm{n}}>6.2 \mathrm{MeV}$, are required. In view of the expected long half-life of $0 v \beta \beta$, a small contribution of a $\gamma$ ray of this energy could be problematic.

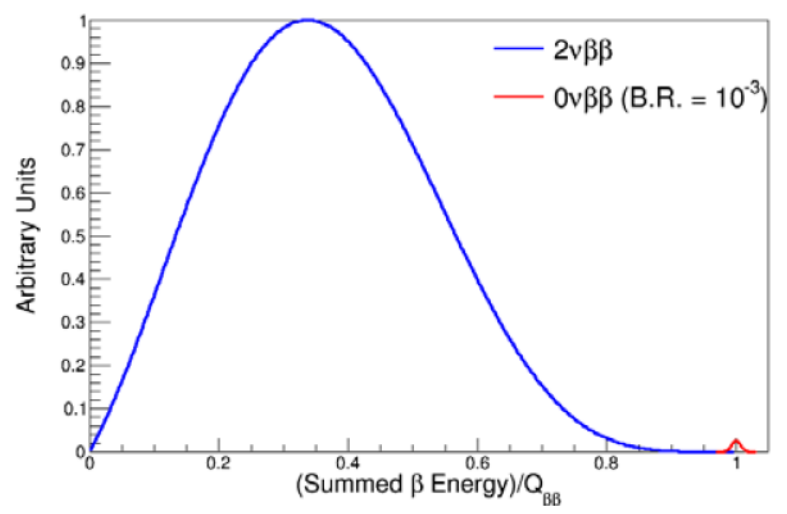

Figure 1. Hypothetical two-neutrino (blue) and neutrinoless (red) double- $\beta$ decay energy spectrum. The resolution and ratio of decay rates (assumed to be $10^{-3}$ in the plot above) affect the potential overlap of the blue and red curves in the region around $\mathrm{Q}_{\beta \beta}[16]$.

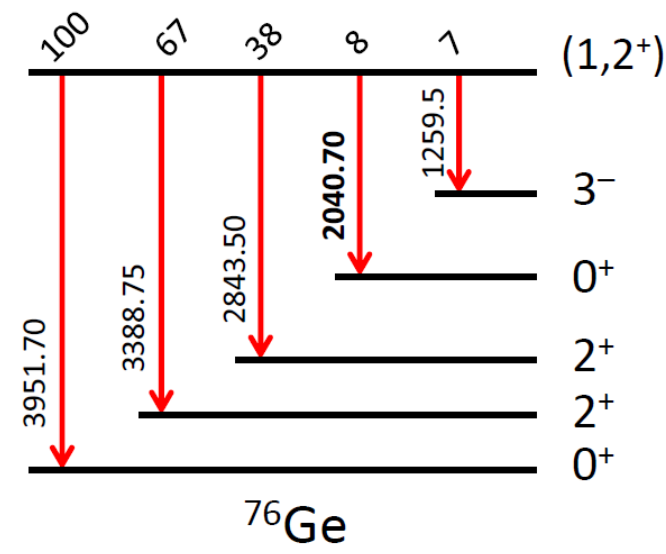

Figure 2. Decays of the $3951.9-\mathrm{keV}$ level of ${ }^{76} \mathrm{Ge}$. The branchings shown are those obtained by Camp and Foster from the $\beta^{-}$decay of ${ }^{76} \mathrm{Ga}[17]$.

Mei and Hime [18] recognized $\gamma$ rays produced from inelastic scattering of cosmic-ray produced neutrons become important for backgrounds in tonne-scale experiments and later assessed background contributions to the continuum from both $\mathrm{Ge}\left(\mathrm{n}, \mathrm{n}^{\prime}\right)$ and $\mathrm{Pb}\left(\mathrm{n}, \mathrm{n}^{\prime}\right)$ and identified a few particularly pernicious $\gamma$ rays $(2041 \mathrm{keV}$ in ${ }^{206} \mathrm{~Pb}$ and a double-escape peak from a $3062-\mathrm{keV}$ transition) from ${ }^{207} \mathrm{~Pb}\left(\mathrm{n}, \mathrm{n}^{\prime}\right)[19]$. Subsequently, Guiseppe et al. [20] performed an investigation of the $\gamma$ rays produced by several $\mathrm{Pb}$ isotopes, which can be found in a $\mathrm{Pb}$-shielded $0 v \beta \beta$ experiment, and measured their cross sections as a function of energy across the range of neutron energies likely to be encountered in the ${ }^{76} \mathrm{Ge}$ experiments. Inelastic neutron scattering studies at GELINA were also carried out to assess background interferences from many materials typically found in 
experimental setups near the $0 v \beta \beta$ experimental signatures of ${ }^{76} \mathrm{Ge}$ and ${ }^{130} \mathrm{Te}[21]$ and, more specifically, on potential background contributors from Ge isotopes [22]. In this latter study, the authors spent a great deal of effort characterizing the contributions to background near $2039 \mathrm{keV}$ from the $3952-\mathrm{keV}$ level in ${ }^{76} \mathrm{Ge}$. Rouki et al. [23] were unable to directly observe the transitions from the 3952-keV level and instead placed limits of a few millibarns. The present measurements address the same questions.

The primary goal of our work was to measure $\left(n, n^{\prime} \gamma\right)$ cross sections for the 3952-keV level over a neutron energy range of 4 to $5 \mathrm{MeV}$ and to establish cross sections for any potential interferences to the $0 v \beta \beta$ experimental signature within this neutron energy range.

\section{Neutron scattering measurements}

\subsection{Nuclear structure measurements}

With the inelastic neutron scattering reaction, nuclear levels can be non-selectively populated up to the incident neutron energy, and lifetimes in the femtosecond regime can be determined. Most low-spin excited states can be observed and transition probabilities can be extracted.

At the University of Kentucky Accelerator Laboratory (UKAL), we have studied the nuclear structure of ${ }^{76} \mathrm{Ge}$ and ${ }^{76} \mathrm{Se}$, the double- $\beta$ decay daughter, with the $\left(\mathrm{n}, \mathrm{n}^{\prime} \gamma\right)$ reaction. Our contributions include an expansion of the low-lying level schemes of these nuclei, measurements of level lifetimes from which transition probabilities can be deduced and compared with model predictions, and the observation of experimental quantities which may affect the actual $0 v \beta \beta$ searches. Complementary photon scattering experiments were conducted on these nuclei with high-intensity bremsstrahlung at the TU Darmstadt and with linearly polarized photons at the High Intensity Gamma Source $(\mathrm{HI} \gamma \mathrm{S})$ at the Duke Free Electron Laser Facility at TUNL. Filling in the gaps in our knowledge of the low-lying levels of these nuclei with new spectroscopic data provides valuable constraints on model descriptions, permitting credible calculations of $M_{0 v}$ 's. In addition to yielding information relevant to $0 v \beta \beta$, these measurements led to new structural insights into ${ }^{76} \mathrm{Se}$ and ${ }^{76} \mathrm{Ge}$, which exhibit intriguing band-like structures and shape coexistence [24].

\subsection{Cross section measurements}

As noted earlier, the $\left(n, n^{\prime} \gamma\right)$ reaction has been identified as an important potential source of background interference in the observation of $0 v \beta \beta$. Given the rarity with which $0 v \beta \beta$ is predicted to occur, knowledge of all interferences in the region of interest is critical. Of particular concern is the aforementioned 3952-keV level in ${ }^{76} \mathrm{Ge}$ [17], with a small ( 4\%) 2041-keV $\gamma$-ray branch that is unlikely to be completely resolved from the $0 v \beta \beta$ experimental signature. Recent studies to measure the cross section of this $\gamma$ ray resulted in an upper limit of 3 $\mathrm{mb}$, but the 2041-keV $\gamma$ ray was not directly observed [23].

Inelastic neutron scattering experiments on enriched samples of ${ }^{76} \mathrm{Ge}$ were performed during two separate runs at UKAL. In both experiments, protons from the $7 \mathrm{MV}$ Van de Graaff accelerator were used to create nearly monoenergetic, fast neutrons via the ${ }^{3} \mathrm{H}(\mathrm{p}, \mathrm{n})^{3} \mathrm{He}$ reaction, which then impinged upon a scattering sample. Two days of beam time during the first data run were devoted to scattering from an elemental ${ }^{76} \mathrm{Ge}$ disc of mass $11.13 \mathrm{~g}$, radius $=1.1 \mathrm{~cm}$, and enriched to $84.12(23) \%$ in ${ }^{76} \mathrm{Ge}$. For the remainder of the data runs, the scattering sample was $41.84 \mathrm{~g}$ of ${ }^{76} \mathrm{GeO}_{2}$ powder with radius $=1.25 \mathrm{~cm}$, height $=4.8 \mathrm{~cm}$, enriched to $85 \%$ in ${ }^{76} \mathrm{Ge}$ and contained within a polyethylene vial. Emitted $\gamma$ rays were detected using a single HPGe detector with an annular BGO shield for Compton suppression.

In the first data run, spectra were accumulated at an incident neutron energy of $4.5 \mathrm{MeV}$ at angles of $50^{\circ}, 90^{\circ}$, and $133^{\circ}$ for the enriched oxide sample and $90^{\circ}$ only for the ${ }^{76} \mathrm{Ge}$ disk. During the second run, data were taken at $125^{\circ}$ for incident neutron energies of $4.3,4.5,4.7$, and 4.9 $\mathrm{MeV}$, as well as additional angles of $65^{\circ}, 147^{\circ}$, and a short $90^{\circ}$ measurement performed for normalization with the first run in an effort to extract angular distribution information. Apart from this last $90^{\circ}$ measurement, each data measurement was performed for 24 hours in order to obtain sufficient population of the 3952-keV level, which has a low cross section. At each incident neutron energy, a $57.00 \mathrm{~g}$ cylindrical sample of natural iron with a radius of $0.95 \mathrm{~cm}$ and a height of $2.54 \mathrm{~cm}$ was employed for normalization of the ${ }^{76} \mathrm{Ge}$ cross sections with ${ }^{56} \mathrm{Fe}$, which is considered a cross section standard. During the first data run, this ${ }^{56} \mathrm{Fe}$ measurement was performed at $90^{\circ}$ at an incident neutron energy of $4.5 \mathrm{MeV}$. A long counter was used to determine the relative neutron fluences when comparing the ${ }^{76} \mathrm{Ge}$ and ${ }^{56} \mathrm{Fe}$ reactions.

\section{Data analysis}

We searched for the production of $\gamma$ rays through the $\left(n, n^{\prime} \gamma\right)$ reaction with monoenergetic accelerator-produced neutrons of 4.0 to $4.9 \mathrm{MeV}$. The three most intense $\gamma$ rays from the $3952-\mathrm{keV}$ level, approximately the $70^{\text {th }}$ excited state, were observed; however, the $2041 \mathrm{-keV}$ branch was not observed with certainty (see Fig. 3 and discussion). From the data obtained and the accepted branching ratios [17], we were able to determine the $\gamma$-ray production cross section at several incident neutron energies, even though the 2041$\mathrm{keV} \gamma$ was below our detection sensitivity. The 3952-keV state has also been excited through the $\left(\gamma, \gamma^{\prime}\right)$ reaction at the TU Darmstadt and at HI $\gamma \mathrm{S}$. At HI $\gamma \mathrm{S}$, excitation with monoenergetic photons often permits a measurement in the absence of interfering backgrounds; however, the 2041-keV branch was below the sensitivity limits.

For the relevant peaks from the $3952-\mathrm{keV}$ level in ${ }^{76} \mathrm{Ge}$, only the three most intense $\gamma$ rays at 2843.50 , 3388.75 , and $3951.70 \mathrm{keV}$ could be fit. There exists at least one additional $\gamma$ ray in the spectra near $2041 \mathrm{keV}$, so this branch cannot be directly determined. This new $\gamma$ ray 
is of considerable interest and will be revisited later. The weakest transition from the 3952-keV level (1259.5 keV) was also found to be obscured by the relatively strong $1259-\mathrm{keV} \gamma$ ray from the $2669-\mathrm{keV}$ level, which is not found in the Nuclear Data Sheets [25] but was recently placed by Toh et al. [2]. The 2844-keV $\gamma$ ray also lies in a region of the spectrum where there are several additional peaks. While it is observed above background, the statistics are meager.

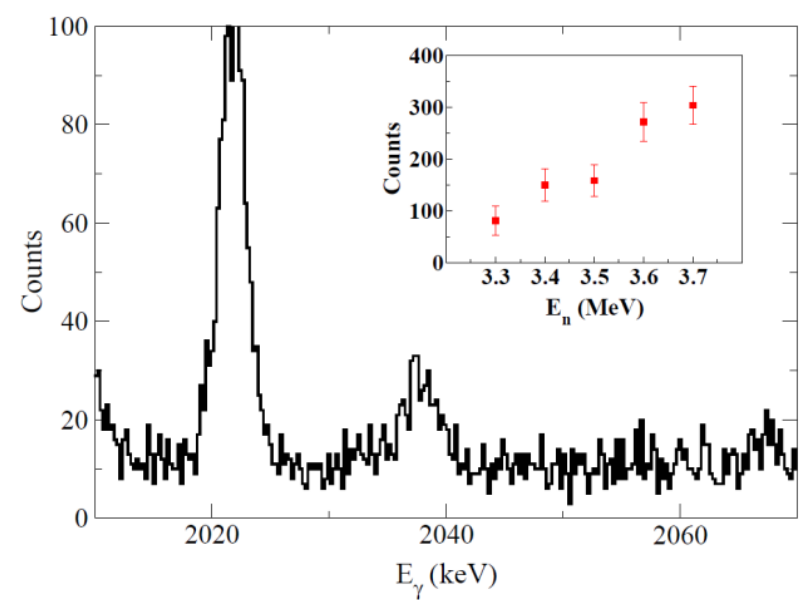

Figure 3. The $2040-\mathrm{keV}$ region of the $\gamma$-ray spectrum from the ${ }^{76} \mathrm{Ge}\left(\mathrm{n}, \mathrm{n}^{\prime} \gamma\right)$ reaction performed at the University of Kentucky Accelerator Laboratory with $3.7 \mathrm{MeV}$ neutrons at a detector angle of $90^{\circ}$. The excitation function for the $2038-\mathrm{keV} \gamma$ ray is shown in the inset.

Energy and detector efficiency calibrations were performed using a ${ }^{226} \mathrm{Ra}$ source. Since the $\gamma$ rays of interest have energies that lie outside of the range of relatively intense ${ }^{226} \mathrm{Ra} \gamma$ rays, extrapolations of the efficiencies and energy nonlinearities were necessary. This procedure is particularly relevant for the efficiency and making this extrapolation warrants an increase in the uncertainties. In addition to energy and efficiency calibrations, other considerations such as incident neutron attenuation, absorption and multiple scattering of $\gamma$ rays, and angular distribution effects where necessary; all were taken into account.

\section{Results and discussion}

From $\left(\mathrm{n}, \mathrm{n}^{\prime} \gamma\right)$ measurements on ${ }^{76} \mathrm{Ge}$ and ${ }^{76} \mathrm{Se}$, a wealth of new spectroscopic information has been obtained, and many new levels were identified and characterized in each nucleus. These data, including level lifetimes, transition probabilities, multipole mixing ratios, and other properties will be provided in a forthcoming publication [24].

In addition, $\gamma$-ray cross sections for the ${ }^{76} \operatorname{Ge}\left(n, n^{\prime} \gamma\right)$ reaction were determined at neutron energies up to 5.0 $\mathrm{MeV}$, with the goal of determining the cross section of $\gamma$ rays in the $2040-\mathrm{keV}$ region, which corresponds to the region of interest in the neutrinoless double- $\beta$ decay of ${ }^{76} \mathrm{Ge}$. Gamma rays from the three strongest branches from the 3952-keV level were observed, but the previously reported $2041-\mathrm{keV} \gamma$ ray was not. Population cross sections of 4 to $7 \mathrm{mb}$ across the range of incident neutron energies were assigned to the $3952-\mathrm{keV}$ level, resulting in a cross section of $\sim 0.1 \mathrm{mb}$ for the $2041 \mathrm{-keV}$ branch using the previously determined branching ratios [17]. Beyond this, the data from these experiments indicate that previously unreported $\gamma$ rays occur in the 2039-keV region.

A new result from this work is the observation of a ${ }^{76} \mathrm{Ge} \gamma$ ray at $2037.5 \pm 0.3 \mathrm{keV}$, which complicates any direct assessment of the previously assigned 2041-keV transition from the 3952-keV level. This new $\gamma$ ray has been assigned to ${ }^{76} \mathrm{Ge}$ from the relative intensities with which it is seen in both the ${ }^{\text {nat }} \mathrm{Ge}$ and ${ }^{76} \mathrm{GeO}_{2}$ samples, where the ratio between the intensities (normalized to the total number of neutrons) is nearly within uncertainty to the ratio of the mass of ${ }^{76} \mathrm{Ge}$ in each of the samples. The same ratio comparison disagrees by at least an order of magnitude for all other Ge isotopes.

This new $\gamma$ ray is much more intense in our spectra than the 20401-keV $\gamma$ ray from the 3952-keV level. Placement of the new $2038-\mathrm{keV} \gamma$ ray is supported by its appearance in the ${ }^{76} \mathrm{Ge}\left(\mathrm{n}, \mathrm{n}^{\prime} \gamma\right)$ excitation function measurements. Unobserved at neutron energies less than 3.1 MeV, the new $\gamma$ ray appears at $3.3 \mathrm{MeV}$ and becomes more intense as the neutron energy increases (see Figure 3 ). As the threshold is between incident neutron energies of 3.1 and $3.3 \mathrm{MeV}$, the level to which this new $\gamma$ ray decays must be the $1108-\mathrm{keV} 2^{+}$level in ${ }^{76} \mathrm{Ge}$. This placement would suggest a new level near $3147 \mathrm{keV}$. Further examination of the excitation function spectra indicates another $\gamma$ ray at $2584.7 \pm 0.3 \mathrm{keV}$ that appears at neutron energies between 3.1 and $3.2 \mathrm{MeV}$. This $\gamma$ ray likely populates the $563-\mathrm{keV} 2^{+}$first excited state from the same $3147-\mathrm{keV}$ level. Figure 4 shows the $\gamma$-ray spectrum at $\mathrm{E}_{\mathrm{n}}=3.7 \mathrm{MeV}$ and the excitation function from 3.2 to $3.7 \mathrm{MeV}$. The $\gamma$ ray to the left in the doublet is the known $2578.55-\mathrm{keV} \gamma$ ray to the first excited state from the $3141.51-\mathrm{keV}$ level.

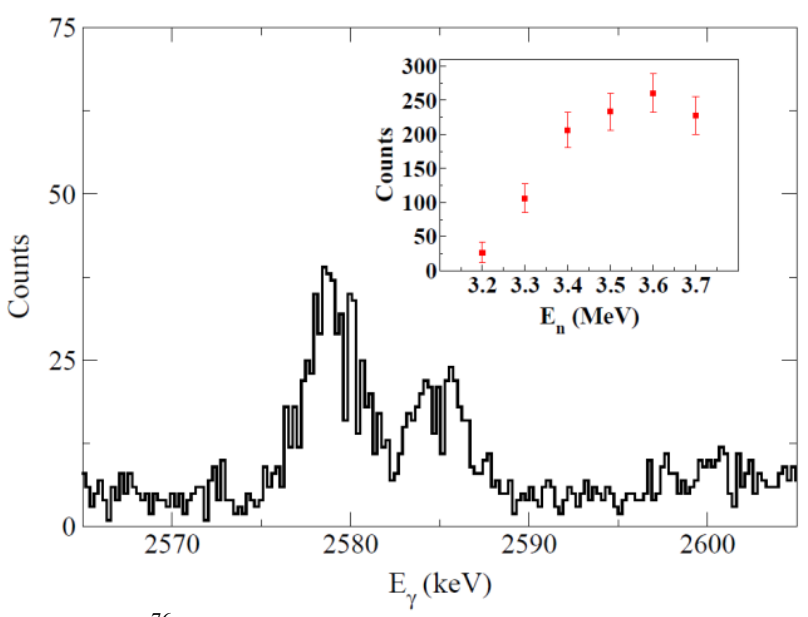

Figure 4. ${ }^{76} \mathrm{Ge}\left(\mathrm{n}, \mathrm{n}^{\prime} \gamma\right)$ spectrum taken at an incident neutron energy of $3.7 \mathrm{MeV}$ and a detection angle of $90^{\circ}$ showing a new $2584.7 \pm 0.3 \mathrm{keV} \gamma$ ray. The inset shows the yield of this $\gamma$ ray at incident neutron energies of 3.2 to $3.7 \mathrm{MeV}$.

If these two new $\gamma$ rays do indeed establish a new level, the lifetimes measured through the Doppler shifts must be similar. From the two data runs for the 2038$\mathrm{keV}$ and $2585-\mathrm{keV} \gamma$ rays, the resultant lifetimes, while not exactly the same, are similar. Unless there are 
missing low-lying levels in ${ }^{76} \mathrm{Ge}$ (a possibility that is not supported by our excitation function data), these two $\gamma$ rays must arise from the same $3147-\mathrm{keV}$ level. The fact that the measured lifetimes are close provides some confirmation of the above. The measurements on the previously known transitions show good agreement, which provides further confidence in the present measurements.

The 2041-keV $\gamma$ ray, which was reported to de-excite the $3952-\mathrm{keV}$ level in ${ }^{76} \mathrm{Ge}$, was not observed in our measurements. As its cross section was once thought crucial for understanding the background in neutrinoless double- $\beta$ decay searches, a detailed study of the $\beta^{-}$decay of ${ }^{76} \mathrm{Ga}$ would prove worthwhile.

\section{Acknowledgements}

The authors gratefully acknowledge the many contributions of H.E. Baber to these measurements. This material is based upon work supported by the U.S. National Science Foundation under Grant No. PHY1305801

\section{References}

1. K. Heyde, J. L. Wood, Rev. Mod. Phys. 83, 1467 (2011)

2. Toh et al., Phys. Rev. C 87, 041304(R) (2013).

3. A. S. Davydov, G. F. Filipov, Nucl. Phys. 8, 237 (1958)

4. T. Nikšić, P. Marević, D. Vretenar, Phys. Rev. C 89, 044325 (2014)

5. L. Wilets, M. Jean, Phys. Rev. 102, 788 (1956)

6. F. T. Avignone, S. R. Elliott, J. Engel, Rev. Mod. Phys. 80, 481 (2008)

7. J. Menéndez, A. Poves, E. Caurier, F. Nowacki, Nucl. Phys. A818, 139 (2009)

8. F. Šimkovic, A. Faessler, H. Müther, V. Rodin, M. Stauf, Phys. Rev. C 79, 055501 (2009)

9. J. Barea, F. Iachello, Phys. Rev. C 79, 044301 (2009)

10. J. Barea, J. Kotila, F. Iachello, Phys. Rev. Lett. 109, 042501 (2012)

11. H. V. Klapdor-Kleingrothaus, A. Dietz, I. V. Krivosheina, O. Chkvorez, Phys. Lett. B586, 198 (2004)

12. M. Agostini, the GERDA Collaboration, Phys. Rev. Lett. 111, 122503 (2013)

13. M. Auger, the EXO Collaboration, Phys. Rev. Lett. 109, 032505 (2012)

14. J. B. Albert, the EXO Collaboration, Phys. Rev. C 89, 015502 (2014)

15. G. Audi, F. G. Kondev, M. Wang, B. Pfeiffer, X. Sun, J. Blanchot, M. MacCormick, Chinese Physics C 36, 1157 (2012).

16. Report to the DOE/NSF Nuclear Science Advisory Committee, Neutrinoless Double Beta Decay, April 24, 2014, p. 19
17. D. C. Camp, B. P. Foster, Nucl. Phys. A177, 401 (1971)

18. D.-M. Mei, A. Hime, Phys. Rev. D 73, 053004 (2006)

19. D.-M. Mei, S. R. Elliott, A. Hime, V. Gehman, K. Kazkaz, Phys. Rev. C 77, 054614 (2008)

20. V. E. Guiseppe, M. Devlin, S. R. Elliott, N. Fotiades, Phys. Rev. C 79, 054604 (2009)

21. A. Hime, D.-M. Mei, R. O. Nelson, D. V. Perepelitsa, Phys. Rev. C 79, 054604 (2009)

22. A. Negret, C. Borcea, A. J. M. Plompen, Phys. Rev. C 88, 027601 (2013)

23. C. Rouki, A. R. Domula, J. C. Drohé, A. J. Koning, A. J. M. Plompen, K. Zuber, Phys. Rev. C 88, 054613 (2013)

24. B. P. Crider, E. E. Peters, T. R. Ross, M. T. McEllistrem, F. M. Prados-Estévez, J. R. Vanhoy, S. W. Yates Phys. Rev. C (to be published)

25. B. Singh, Nucl. Data Sheets 74, 63 (1995). 
